\title{
Analysis on the Phase Frequency Characteristic of Soil Impedance
}

\author{
Xiaobin Cao, Zhongmei Li, Shiwei Yao \\ School of Electrical Engineering, Southwest Jiao Tong University, Chengdu, China \\ Email: 834900889@qq.com
}

How to cite this paper: Cao, X.B., Li, Z.M., and Yao, S.W. (2018) Analysis on the Phase Frequency Characteristic of Soil Impedance. Energy and Power Engineering, 10, 17-24.

https://doi.org/10.4236/epe.2018.104B003

Received: January 29, 2018

Accepted: April 8, 2018

Published: April 11, 2018

\begin{abstract}
Soil is a typical porous media and its impedance characteristic directly determines the performance of grounding system. Soil phase frequency characteristic measurements were carried out on various soil types and water content. This paper finds that the impedance angle of soil specimen presents a capacitive performance when power frequency $(f)$ is low. As the frequency increases, soil impedance angle goes up rapidly. Furthermore the frequency characteristic while $f>1000 \mathrm{~Hz}$ is distinct in terms of different water content. In particular, at low moisture content, soil impedance angle would be higher than $0^{\circ}$, that is, the inductive component is obvious. The study result indicates that porous media possesses the unique conductivity property different from conductor and solution. Its mechanism needs further study.
\end{abstract}

\section{Keywords}

Soil Impedance Angle, Frequency Characteristic, Porous Media, Water Content, Particle Size

\section{Introduction}

Soil, as a typical porous material is the major factor impacting impedance characteristic of grounding system [1]. Therefore soil is compatibly selected as a representative sample for studying the conductivity characteristic of porous media. Porous material is widely applied in grounding, capacitor and battery, but previous papers on the electrical properties of porous media are rare [2] [3]. The solid particles in soil consist of the skeleton and pore structure of porous medium, and the pore water forms the primary medium for electric conduction [4] [5]. Some previous papers have analyzed the measurement method of soil resistivity [6] [7]. In the research of soil impulse impedance characteristic, it was found that frequency-dependent characteristic as an obvious influence on the 
impulse performance in soil [8] [9]. The main focus of researches in existence is the amplitude frequency characteristic of soil impedance, while this paper studies the frequency characteristic of soil impedance angle under various soil types and water content. Consequently, this paper reveals the unique conductivity property of porous media.

\section{Experiment Mode}

\subsection{Preparation of Soil Specimen}

According to the measurement method of dielectric impedance characteristic, this paper adopts the cube specimen box shown in Figure 1. Four flanks of the cube box with $15 \mathrm{~cm}$ side length are made of plexi glass material. The top and bottom surface are both the current pole made of red copper. The top surface is composed of two kinds of electrodes. The middle electrode as the electrode for measuring current with a size of $10 \mathrm{~cm} \times 14 \mathrm{~cm}$ and the auxiliary two on both sides with $1.5 \mathrm{~cm} \times 14 \mathrm{~cm}$ in size are parallel connected to the end of power source aimed to eliminate the influence of specimen edge. The $15 \mathrm{~cm} \times 15 \mathrm{~cm}$ bottom electrode connected to the other end serves as a common electrode for the circumfluence of experimental current.

For avoiding interference, Four-electrode Method is adopted to test the specimen impedance in the experiment. In Figure 1, Pole $1\left(P_{1}\right)$ and Pole $2\left(P_{2}\right)$ are the voltage measurement probe. The distance from $P_{1}$ to the common electrode $I_{1}=10.5 \mathrm{~cm}$ and that from $P_{2} l_{2}=4.5 \mathrm{~cm}$ are fixed, and the space between $P_{1}$ and $P_{2}$ equals $6 \mathrm{~cm}$.

\subsection{Experiment Circuit}

Figure 2 presents the experiment circuit. The $9 \mathrm{k} \Omega R_{1}$ is used as a current limiting resistor. The $R_{2}$, equal to $1 \mathrm{k} \Omega$, is a non-inductive resistance regarded as the sampling resistance for circuit current. Moreover, the voltage between the two measuring poles $\left(P_{1}\right.$ and $\left.P_{2}\right)$ is also taken by oscilloscope. The applied voltage of $6 \mathrm{~V}$ amplitude lasts for no exceeding $1 \mathrm{~min}$.

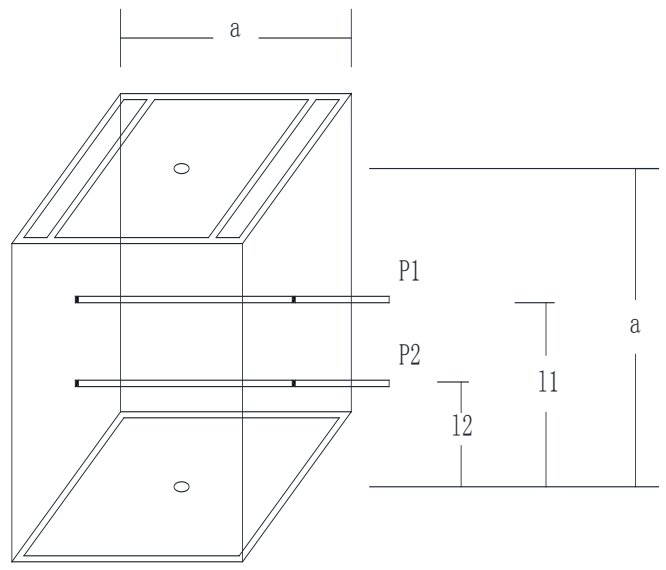

Figure 1. Framework of soil specimen box. 


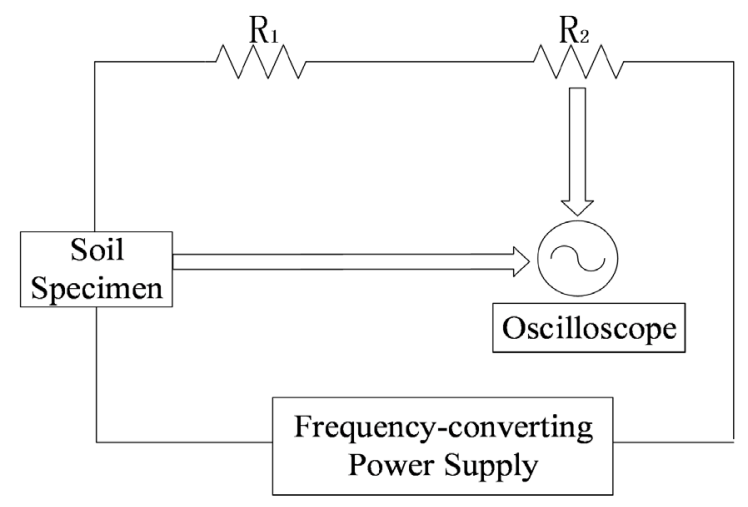

$\mathrm{R}_{1}$; Current limiting resistance; $\mathrm{R}_{2}$ : Non-inductive sampling resistance

Figure 2. Experiment circuit.

Therefore, the current amplitude of soil specimen can be calculated based on the resistance of $R_{2}$. The soil impedance phase ( $\varphi$ ) will be obtained by comparing the phase difference between the specimen voltage and current measured on oscilloscope and calculated as following:

$$
\varphi=\varphi_{U}-\varphi_{i}
$$

where $\varphi_{U}$ is the voltage phase, $\varphi_{i}$ is the current phase.

\subsection{Measurement on Water Content, Density and Porosity in Soil}

The paper chooses three Chinese common soil, yellow soil, latosolic red soil and red soil, as the experimental specimen. The collected soil is filled into the cube containers that are firstly weighted. And then soil specimen is gently compacted, smoothed and weighted. Ultimately, water is gradually sprinkled into the specimen accompanied 30-min stationary at least. It is considered that specimens are saturated with water and suitable for weighing until the water in soil surface no longer permeates. Then other experimental units are prepared by the same means above.

When water content in soil sample needs to be changed, test units as a whole is taken into the $150^{\circ} \mathrm{C}$ drying box for weight reduction and stand for two days for even water distribution. The specimen quality at present weighs $m_{l}$. Samples are again put into the oven to bake and reduce weight after each experiment. Repeat the operations above until the test unit weight no longer change after several repeated experiments. At the point, this paper considers the water content of dried soil equals to 0 and records the sample weight as $m_{0}$. The water content $k$, soil density $\rho$, porosity $P$ are respectively can be calculated by equation (2), (3) and (4).

$$
\begin{gathered}
k=\frac{m_{1}-m_{0}}{m_{0}} \times 100 \% \\
\rho=\frac{m_{0}}{V-V_{w}}
\end{gathered}
$$




$$
P=\frac{V_{w}}{V}
$$

where $V$ is the soil volume before backing, $V_{w}$ is the volume of water when soil is saturated with water. Parameters on three soil samples are illustrated in Table 1.

In Agrology, soil particle size can be judged referring to soil porosity or density. In a same volume specimen, soil with high porosity or small density possesses a relatively larger particle size and moisture content. Comparing the data in Table 1, the particle size of the three soil rates from small to large is red soil, latosolic red soil and yellow soil.

\section{Frequency Characteristic under Different Influence Factors}

\subsection{Impact of Various Water Content}

The experiment result on frequency characteristic of yellow soil impedance angle at six different moisture content is indicated in Figure 3. The impedance angle of soil specimen keeps less than $0^{\circ}$ when water content is higher $(k \geq 7.17 \%)$. As the frequency increases, the impedance angle will generally approach $0^{\circ}$. That is soil impedance is initially capacitive, and gradually shifts to resistivity with frequency rising.

Table 1. Parameters of three soil.

\begin{tabular}{cccc}
\hline & Yellow Soil & Latosolic Red Soil & Red Soil \\
\hline$\rho /\left(\mathrm{g} / \mathrm{cm}^{3}\right)$ & 1.86 & 1.75 & 1.72 \\
$P / \%$ & 39.1 & 47.7 & 49.1 \\
\hline
\end{tabular}

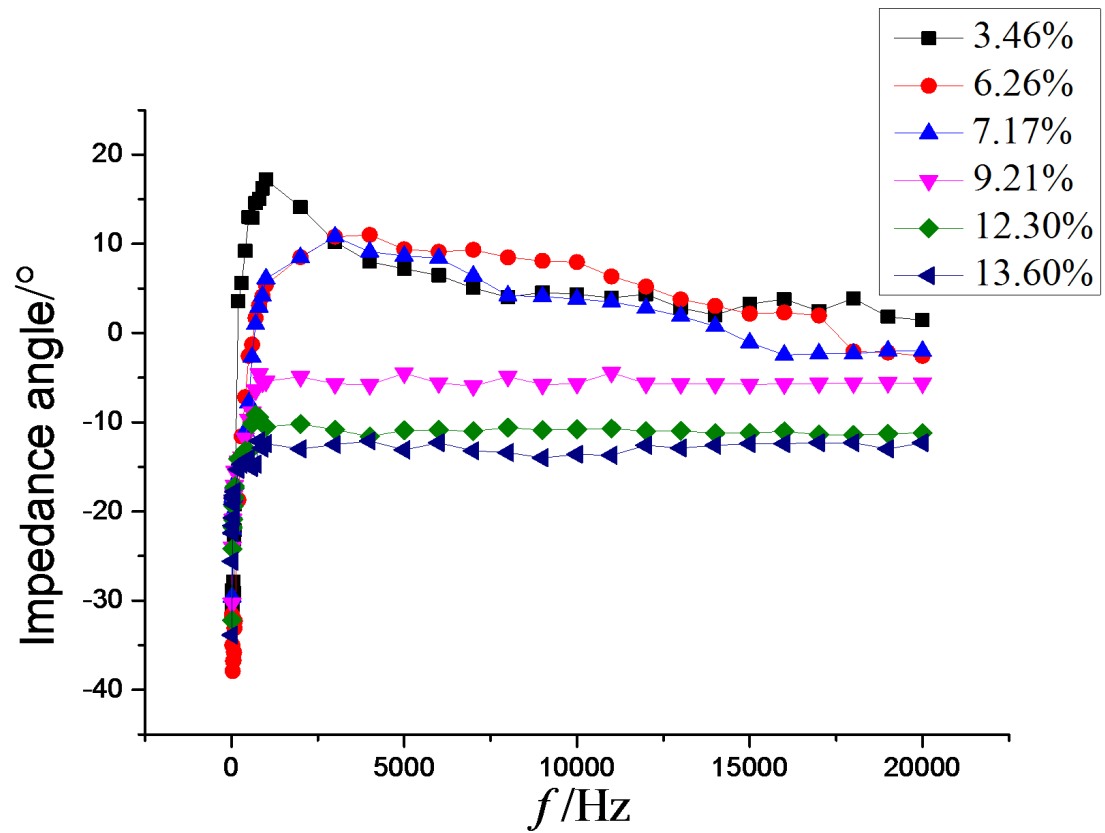

Figure 3. Phase frequency characteristic at various water content. 
While $k<7.17 \%, f<500 \mathrm{~Hz}, \varphi$ goes up from less than $0^{\circ}$ to the greater with the raise of frequency, which means soil impedance has changed into inductance. Nevertheless further frequency increment will make $\varphi$ decrease to $0^{\circ}$. Specimen impedance becomes resistive from inductive.

\subsection{Impact of Different Particle Sizes}

The same method is utilized to respectively test yellow soil, latosolic red soil and red soil. The phase frequency characteristic of soil specimen with three particle sizes at different water saturated levels is shown in Figure 4.

As illustrated in Figure 4, particle size has a negligible effect on the impedance angle. It can be considered that the phase frequency characteristic of soil impedance is approximately independent of soil particle size. No matter what type of soil, their phase frequency characteristic is common. On the other hand, the impedance angle of soil specimen with different water content processes similar variation law in the low frequency range. While the frequency exceeds $1000 \mathrm{~Hz}$, soil impedance angle gradually tends to a certain valued determined by water content.

\section{Analysis on the Phase Frequency Characteristic of Soil Impedance}

\subsection{Frequency Characteristic of Soil Impedance Angle When $f<1000 \mathrm{~Hz}$}

This paper takes yellow soil at various water saturated levels as an example. The phase frequency characteristic when $f<1000 \mathrm{~Hz}$ is shown in Figure 5. Frequency

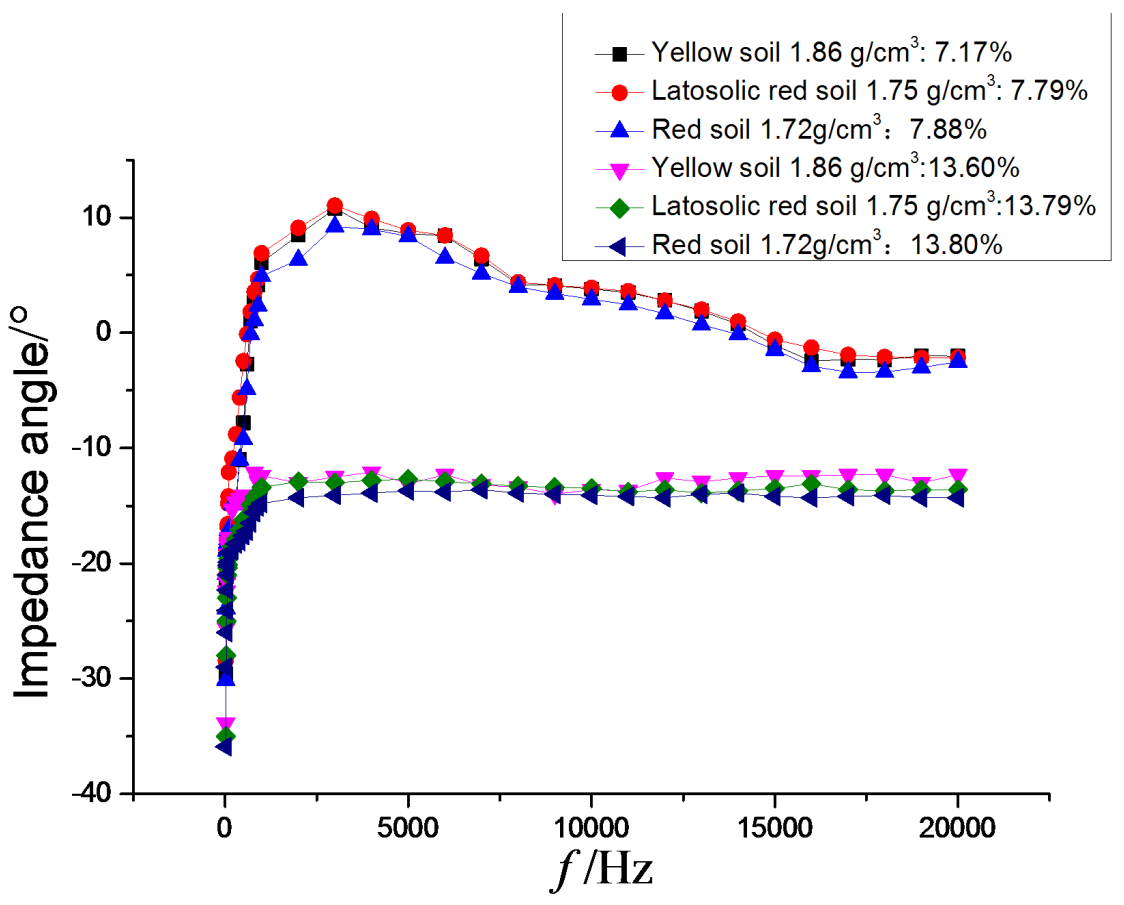

Figure 4. Phase frequency characteristic with different particle sizes. 


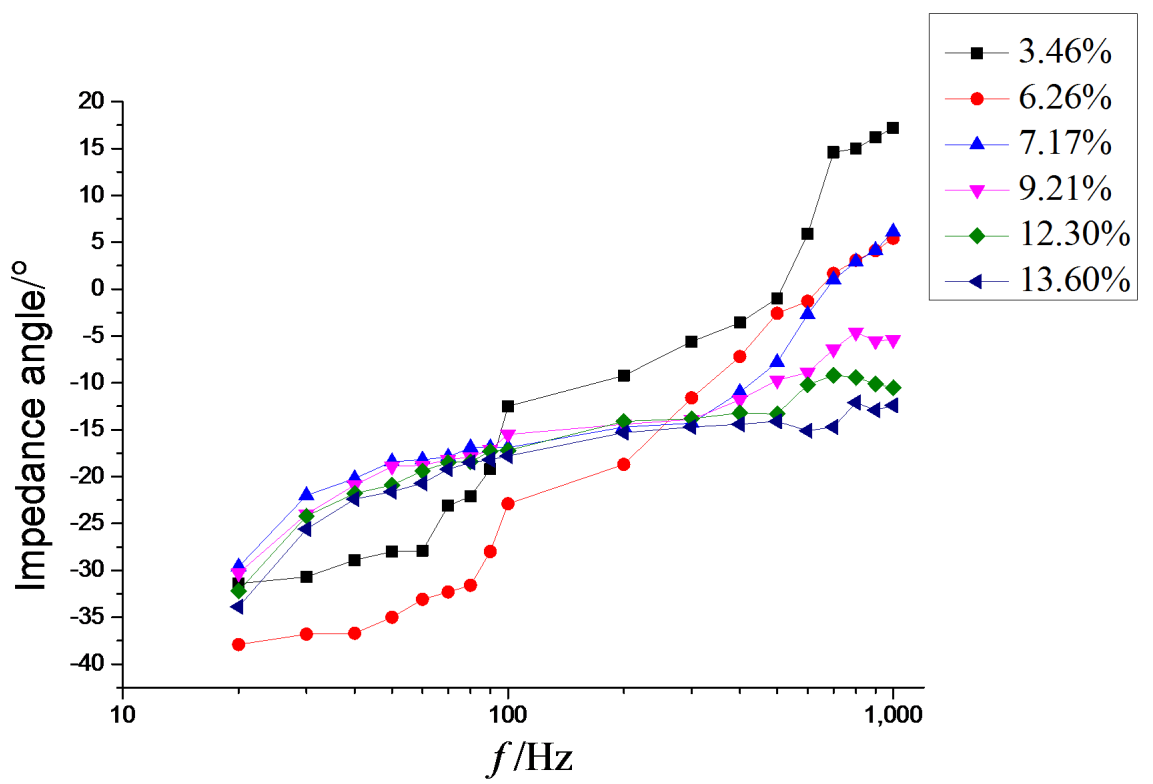

Figure 5. Phase frequency characteristic when $f<1000 \mathrm{~Hz}$.

is expressed by the logarithmic derivative. The relationship between impedance angle and the frequency logarithm is approximately proportionate. The variation amplitude of the impedance angle at low moisture content is greater than that at the high. For example, when $k=3.46 \%$, phase span reaches $49^{\circ}$; while $k=$ $13.60 \%$, impedance angle range is $-35^{\circ}-13^{\circ}$ with $22^{\circ}$ phase span.

Figure 6 presents that soil conduction is mainly formed by the movement of ionic conductors in pore water. Ions under electric field move after being applied different frequency power source at both ends of soil specimen: the positive ions move toward to the cathode, and the negative is versa. The deviation of the gravity center between the positive and negative ions results from ionic movement in soil, resulting in soil dielectric migration polarization and capacitance effect.

\subsection{Frequency Characteristic of Soil Impedance Angle When $f>1000 \mathrm{~Hz}$}

The experimental results at high frequency are indicated in Figure 7. When $k$ is high enough, $\varphi$ would be less than $0^{\circ}$. As the level saturated with water goes up, impedance angle decreases and the capacitance component of soil impedance is enhanced.

While $k$ is relatively low, $\varphi$ would be more than $0^{\circ}$, which means that soil impedance contains inductive component. As the frequency further rises, the soil impedance angle gradually decreases, that is inductive component gradually weakens and eventually converts into capacitive characteristic.

As a typical porous material, soil is composed of solid skeleton and pore space saturated with water. It depends on ion migration in pore water to form electric current, so current path is affected remarkably by pore water content. Under the 


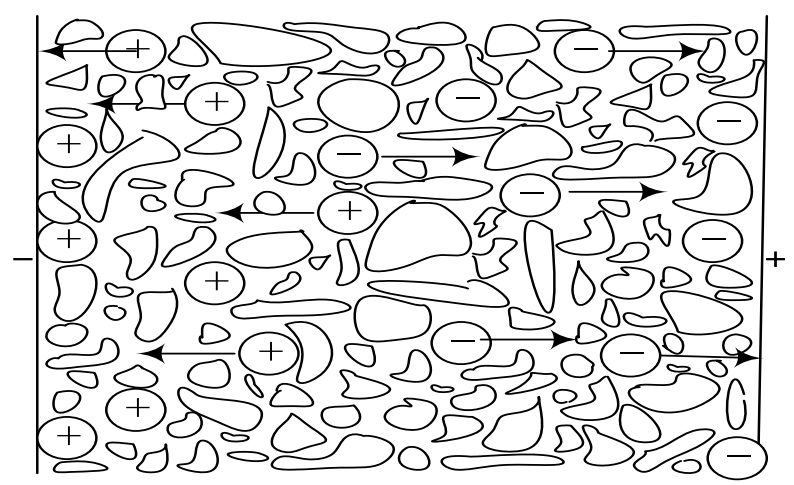

Figure 6. Ion movement inside soil.

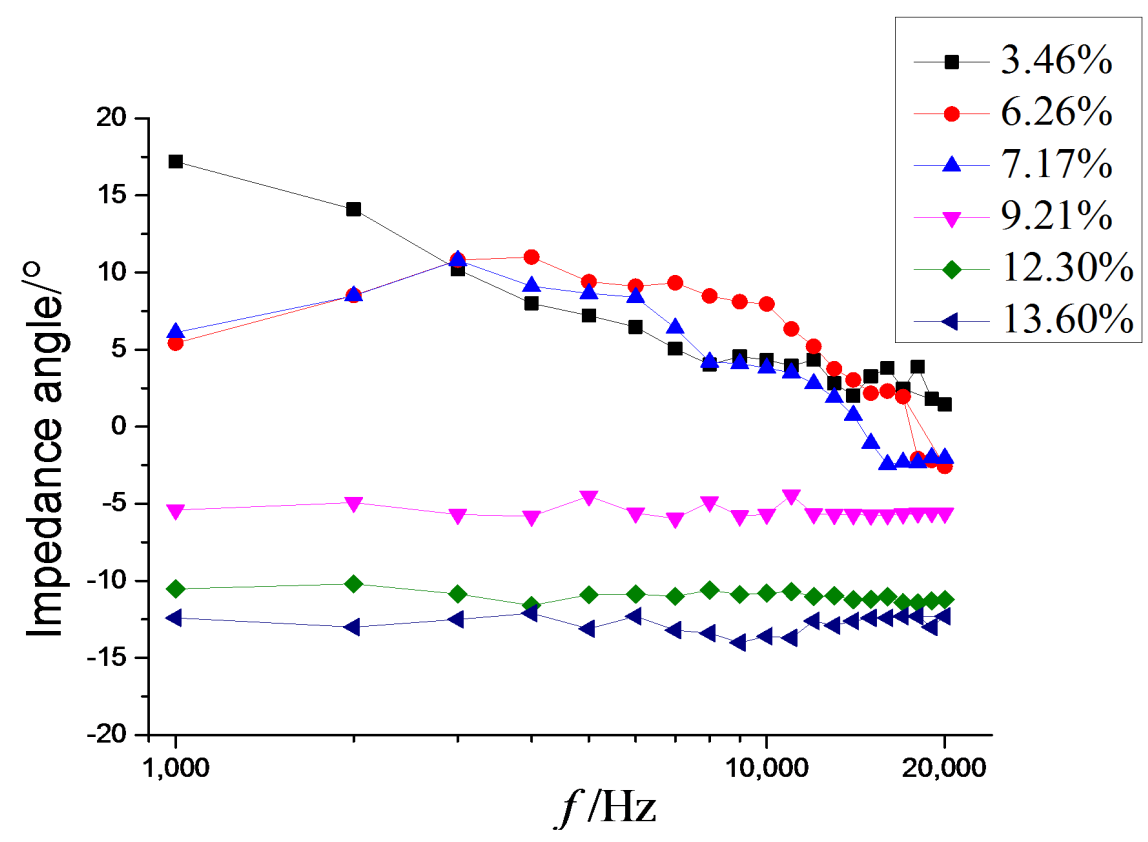

Figure 7. Phase frequency characteristic when $f<1000 \mathrm{~Hz}$.

action of magnetic field produced by ion movement, the inductance and mutual inductance of pore channel in soil will produce inductive component to soil impedance. Therefore, the phase frequency characteristic of soil impedance is the interaction between capacitive component and inductive component.

\section{Conclusion}

The paper studied the phase frequency characteristic of soil impedance based on the experimental circuit above. Soil impedance angle changes regularly with the frequency of external power source. Soil impedance performs capacitive characteristic when $f<1000 \mathrm{~Hz}$. As the frequency increases, the impedance angle rapidly goes up to around $0^{\circ}$. Namely, soil specimen impedance presents the variation law from capacitive to resistive impedance. While $f>1000 \mathrm{~Hz}$, water content will obviously impact the variation law with frequency. At the low water saturated level, the phase angle may be higher than $0^{\circ}$, but eventually decreases to 
$0^{\circ}$ as the frequency rises, which means the overall impedance has become inductive. Furthermore the particle size has a negligible effect on the phase frequency characteristic of soil impedance.

\section{Acknowledgements}

This study was supported by National Natural Science Foundation of China (No: 51777175).

\section{References}

[1] Xing, P., Lu, H., Tong, X., et al. (2016) Research on Influence of Soil Characteristic on Impulse Grounding Resistance of Substation Grounding Grid. Engineering Journal of Wuhan University, 49, 223-228.

[2] Wang, P., Wang, H. and Zhang, J. (2014) Design and Application of Supercapacitor Energy Storage System Used in Low Voltage Ride through of Wind Power Generation System. Proceedings of the CSEE, No. 10, 1528-1537.

[3] Zhao, W., Zhu, H. and Chen, Y. (2011) Research on the Movement Law of Positive Ions and Electrons in the Plasma Channel of EDM. IEEE.

[4] Cao, X., Wu, G., Fu, L., et al. (2007) Study of the Temperature Impact on Soil Resistivity. Transactions of China Electrotechnical Society, 22, 1-6.

[5] Yuan, R. (2011) Study on Fluid Flow Passing through a Porous Media. Acta Analysis Functionalis Applicata, 13, 357-365.

[6] Choi, J.H. and Lee, B.H. (2012) An Analysis on the Frequency-Dependent Grounding Impedance Based on the Ground Current Dissipation of Counterpoises in the Two-Layered Soils. Journal of Electrostatics, 70, 184-191. https://doi.org/10.1016/j.elstat.2011.12.003

[7] Zhu, B. (2015) Study on the Variation Characteristic of Soil Electrical Parameters and the Influence on Grounding Device's Performance. Chongqing University, Chongqing.

[8] Sima, W., Tang, Y., Yuan, T., et al. (2015) Influence of Soil Dielectric Constant's Frequency-Dependent Characteristic on Impact Current Dispersal of Grounding Electrode. High Voltage Engineering, 41, 1512-1518.

[9] Visacro, S., Alipio, R., Murta Vale, M.H., et al. (2011) The Response of Grounding Electrodes to Lightning Currents: The Effect of Frequency-Dependent Soil Resistivity and Permittivity. IEEE Transactions on Electromagnetic Compatibility, 53, 401-406. https://doi.org/10.1109/TEMC.2011.2106790 